\title{
Heterogeneity Wavelet Kinetics from DCE-MRI for Classifying Gene Expression Based Breast Cancer Recurrence Risk
}

\author{
Majid Mahrooghy, Ahmed B. Ashraf, Dania Daye, Carolyn Mies, Michael Feldman, \\ Mark Rosen, and Despina Kontos
}

The University of Pennsylvania, Philadelphia, PA 19104, USA

Majid.Mahrooghy@uphs. upenn.edu

\begin{abstract}
Breast tumors are heterogeneous lesions. Intra-tumor heterogeneity presents a major challenge for cancer diagnosis and treatment. Few studies have worked on capturing tumor heterogeneity from imaging. Most studies to date consider aggregate measures for tumor characterization. In this work we capture tumor heterogeneity by partitioning tumor pixels into subregions and extracting heterogeneity wavelet kinetic (HetWave) features from breast dynamic contrast-enhanced magnetic resonance imaging (DCE-MRI) to obtain the spatiotemporal patterns of the wavelet coefficients and contrast agent uptake from each partition. Using a genetic algorithm for feature selection, and a logistic regression classifier with leave one-out cross validation, we tested our proposed HetWave features for the task of classifying breast cancer recurrence risk. The classifier based on our features gave an ROC AUC of 0.78 , outperforming previously proposed kinetic, texture, and spatial enhancement variance features which give AUCs of $0.69,0.64$, and 0.65 , respectively.
\end{abstract}

Keywords: Breast DCE-MRI, breast tumor heterogeneity partitioning, tumor feature extraction, breast cancer recurrence prediction.

\section{Introduction}

Breast dynamic contrast enhanced magnetic resonance imaging (DCE-MRI) has evolved into an effective modality for detection, prognostic assessment, diagnosis, prediction of response to therapy, and characterization of breast tumors [1]. In DCEMRI the patient is first administered a contrast agent and then a temporal sequence of MRI images is acquired. By capturing the spatiotemporal patterns of contrast agent uptake, DCE-MRI can be used as complementary to mammography and ultrasonography. Several studies have been performed to obtain diagnostic, prognostic, or predictive markers using DCE-MRI for characterizing the breast tumors in terms of benign, malignant, invasive ductal carcinoma (IDC), ductal carcinoma in situ (DCIS), lymph node involvement, tumor grade, and other histopathologic markers $[2,3]$.

Different algorithms and methodologies have been developed using DCE-MRI to provide quantitative markers for characterizing lesions [3-5]. Kinetic, morphological, 
textural, and spatio-textural are among the most commonly used features extracted from DCE-MRI. The kinetic features usually include maximum uptake, peak location, and wash-out rate [2]. Chen et. al. [3] developed spatial enhancement variance features and used them along with kinetic and morphological features to distinguish between the malignant and benign breast lesions. Lee et. al. [4] obtained the spatiotemporal features from three levels using pixel-wise kinetic features, the spatial features from the kinetic features, and extracting kinetic association of spatial features. Zheng et. al. [5] developed spatiotemporal features using temporal enhancement by applying the Fourier transform and employing Gabor filter and moment invariant descriptors. There are other methods which developed spatiotemporal features using textural kinetics [6]. Although useful, a limitation of these methods is that the spatiotemporal features are not based on heterogeneity partitioning and also they do not consider the enhancement rate of spatial frequency.

Breast tumors have been shown to be heterogeneous lesions, and tumor heterogeneity is one of the major difficulties in the way of an effective cancer diagnosis and successful treatment [7]. In DCE-MRI images, the tumor pixels act heterogeneously both spatially and temporally. As such, finding appropriate kinetic features that capture tumor heterogeneity is challenging. Traditional DCE-MRI analysis methods have mainly focused on aggregate kinetic measures [3-6]. However, aggregate kinetic measures fall short of capturing the intra tumor heterogeneity which is a crucial aspect of tumor characterization [7]. In this regard, we propose a tumor partitioning scheme using entropy and intensity variance as a measure of heterogeneity. We also define new features which consider both the statistical and spatial frequency of the partitioned area with respect to the temporal data. Obtaining multi-resolution kinetic information from tumors in DCE-MRI at different spatial frequencies and the rate of the frequency change over time may provide more relevant features to characterize the heterogeneity in the spatiotemporal patterns of tumor enhancement, which can lead to better performance in detection, prognosis, and prediction. We test our proposed features using a genetic algorithm wrapper based feature selection [8] for classifying women diagnosed with breast cancer into low and high risk of cancer recurrence which is critical in making treatment decisions [9].

\section{Methods}

We first partition the DCE-MRI images into different tumor sub-regions in which we further capture the heterogeneity of pixels. Heterogeneity wavelet kinetic (HetWave) features are then extracted from the partitions to obtain the spatiotemporal patterns of contrast agent uptake as well as spatial frequency information using wavelet coefficients from the partitions. To reduce the dimensionality and also avoid classifier overfitting, a genetic algorithm wrapper based feature selection scheme is used. The selected features are used to learn a logistic regression model for classifying breast cancer recurrence risk. A leave-one-out cross validation is employed to evaluate classifier generalization. Figure 1 shows a block diagram of the proposed features and the algorithm. 


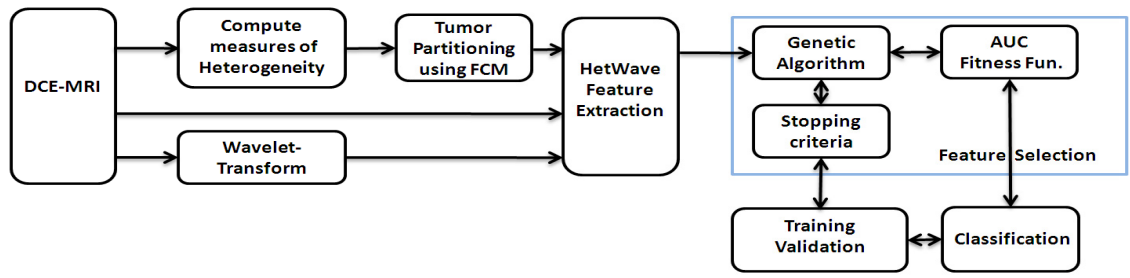

Fig. 1. Block diagram of tumor HetWave feature extraction and recurrence risk classification

\subsection{Heterogeneity Partitioning}

We propose to divide the tumor into different clusters using entropy and intensity variance as a measure of heterogeneity. We use a sliding window $(5 \times 5$ pixels $\approx 4$ $\mathrm{mm} 2$ ) and compute the entropy and variance of the pre-contrast intensity values within the sliding window. We obtain these two features for each pixel and then apply a step of fuzzy C-mean (FCM) clustering to the features to have three partitions (i.e., high, medium, and low degree of heterogeneity which are sorted according to $\mathrm{L}_{2}$ norm of their centroid). Figure 2 shows the input image and the output partitioning for two examples of high and low recurrence risk tumors. The output of this step is a mask image $\mathrm{M}$ such that $\mathrm{M}_{\mathrm{k}}$ represents the partition number of the k-th pixel.

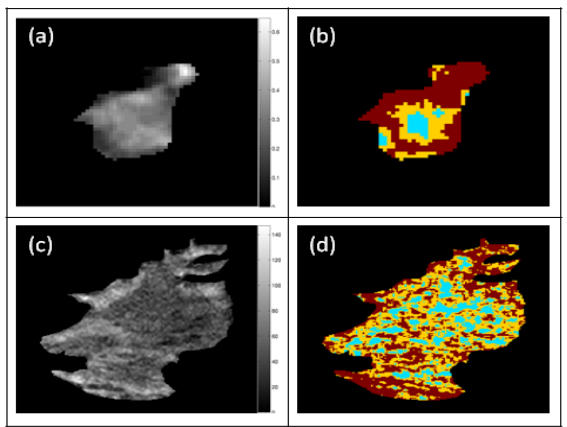

Fig. 2. (a) Pre-contrast image of a low recurrence risk lesion; (b) the corresponding heterogeneity partitioning of the low risk tumor (red, orange, and blue show the high, medium, and low heterogeneity measure (i.e. clusters), respectively); (c) Pre-contrast image of a high recurrence risk lesion; (d) the corresponding heterogeneity partitioning of the high risk tumor

\subsection{Heterogeneity Wavelet Kinetic (HetWave) Features}

To capture the spatial frequency information, we use the wavelet transform [10]. The output of the wavelet transform includes the coefficients for different spatial frequencies at multiple resolutions. Specifically, the image is low pass filtered and downsampled to give the approximation coefficients [10]. The image is also high pass filtered to give three detail coefficients corresponding to horizontal, vertical, and diagonal spatial frequencies. We obtain two level decompositions (in the rest of the paper, they 
are referred to as DP1 and DP2) of the wavelet transform (Haar wavelet family) for every pre- and post-contrast image. Since each decomposition image is down-sampled from the original image, the corresponding tumor mask is also dowsampled to match it with the wavelet images. We then extract the mean and variance of the approximation and detail (horizontal, vertical, and diagonal) images at DP1 and DP2 within each partition. In addition, we extract the mean and variance of pre- and post-contrast images within each partition. In the following we give the mathematical definitions for our HetWave features:

Let the pre- and post-contrast images be defined as $I_{t}$ where $t \epsilon\{0,1,2,3\}$. As such the approximate, $C_{L}^{t}(\mathrm{k})$, and detail coefficients, $D_{L}^{t, s}(\mathrm{k})$, can be computed as follows [10]:

$$
\begin{gathered}
C_{L}^{t}(\mathrm{k})=<I_{t}, \Phi_{L, k}> \\
D_{L}^{t, s}(\mathrm{k})=<I_{t}, \psi_{L, k}^{S}>
\end{gathered}
$$

where, $\left\langle\mathbf{x}, \mathbf{y}>\right.$ represents the inner product between the vectors $\mathbf{x}$ and $\mathbf{y}$, and $I_{t}, \Phi_{L, k}$, and $\psi_{L, k}^{S}$ are the vectorized versions of the image, the scaling function, and the wavelet function respectively [10]. In the above equation, the variable $k$ is used to index the pixels in the vectorized images, and $L$ represents the decomposition level. The coefficients of $D_{L}^{t, s}$ can be horizontal, vertical, and diagonal detail ( $\left.s=H, V, D\right)$ [10]. Illustrations of representative wavelet coefficients are shown in Figure 3.

As mentioned in Section 2.1 above, $M$ is the mask capturing pixel partitioning such that $M_{k}$ represents the membership mapping of pixel $k$ to its respective partition, where $M_{k} \in\{1,2,3\}$. We then compute the mean and variance of the approximation and detail coefficients (horizontal, vertical, and diagonal) at DP1 and DP2 for each partition as follows:

Assuming $\mu_{C A, L}$ to be the mean of approximation coefficients at decomposition level $\mathrm{L}$ and $\mu_{c H, L}, \mu_{C V, L}$, and $\mu_{c D, L}$ to be the mean of detail coefficients (horizontal, vertical, and diagonal), we have: (since the formula of $\mu_{c V, L}$ and $\mu_{c D, L}$ are similar to $\mu_{c H, L}$, we only show $\mu_{c H, L}$ for simplicity purpose).

$$
\begin{aligned}
\mu_{c A, L}(i, t) & =\frac{\sum_{k=1}^{N} C_{L}^{t}(k) \delta\left(M_{k}=i\right)}{\sum_{k=1}^{N} \delta\left(M_{k}=i\right)} \\
\mu_{c H, L}(i, t) & =\frac{\sum_{k=1}^{N} D_{L}^{t, H}(k) \delta\left(M_{k}=i\right)}{\sum_{k=1}^{N} \delta\left(M_{k}=i\right)}
\end{aligned}
$$

where $\delta\left(M_{k}=i\right)$ is an indicator function that equals 1 when $M_{k}=i$, and zero otherwise; $i$ represents the partition number, and $N$ is the total number of tumor pixels.

We also can compute the variance for approximation $\left(\sigma_{c A, L}^{2}\right)$ and detail coefficients $\left(\sigma_{c H, L}^{2}, \sigma_{c V, L}^{2}\right.$, and $\left.\sigma_{c D, L}^{2}\right)$ as follows:

$$
\begin{aligned}
\sigma_{c A, L}^{2}(i, t) & =\frac{\sum_{k=1}^{N}\left(C_{L}^{t}(k)-\mu_{c A, L}(i, t)\right)^{2} \delta\left(M_{k}=i\right)}{\sum_{k=1}^{N} \delta\left(M_{k}=i\right)} \\
\sigma_{c H, L}^{2}(i, t) & =\frac{\sum_{k=1}^{N}\left(D_{L}^{t, H}(k)-\mu_{c H, L}(i, t)\right)^{2} \delta\left(M_{k}=i\right)}{\sum_{k=1}^{N} \delta\left(M_{k}=i\right)}
\end{aligned}
$$

In addition to the above statistics of wavelet coefficients, we obtain the mean and variance of the pre- and post-contrast images $\left(I_{t}\right)$ within each partition. 


$$
\begin{gathered}
\mu_{I}(i, t)=\frac{\sum_{k=1}^{N} I_{t}(k) \delta\left(M_{k}=i\right)}{\sum_{k=1}^{N} \delta\left(M_{k}=i\right)} \\
\sigma_{I}^{2}(i, t)=\frac{\sum_{k=1}^{N}\left(I_{t}(k)-\mu_{I}(i, t)\right)^{2} \delta\left(M_{k}=i\right)}{\sum_{k=1}^{N} \delta\left(M_{k}=i\right)}
\end{gathered}
$$

For every image sequence, there are 3 partitions and 2 statistics (mean/variance). As such there are 6 statistics associated with each time-point for an image sequence. After computing the above statistics of wavelet coefficients within each partition, we compute the signal enhancement ratio (SER) of each statistic. Hylton [11] proposed the signal enhancement ratio (SER) such that $S E R=\left(I_{1}-I_{0}\right) /\left(I_{2}-I_{0}\right)$ where $I_{0}$, $I_{1}$, and $I_{2}$ represent the signal intensities on the precontrast, early postcontrast, and late postcontrast, respectively. We define HetWave, motivated by SER, where instead of using intensity, we use the 6 statistics explained above for each time point as follows:

$$
\text { HetWave }_{f}=\frac{W_{1}^{f}-W_{0}^{f}}{W_{3}^{f}-W_{0}^{f}}
$$

where $W_{\mathrm{t}}^{f}$ represents the statistics defined above and $(f \in\{1, \ldots, 6\})$ for the contrast image corresponding to the time point $t \in\{0,1,2,3\}$ ( 0 is the pre-contrast and 1,2 , and 3 are the post-contrast images).

In summary, we have 4 wavelet images (i.e., approximate, horizontal, vertical, diagonal) for each decomposition level (i.e., for 2 decomposition levels we would have 8 wavelet images). We also compute statistics for the input image sequence. As such, in all we have 9 image sequences, and 6 HetWave features for each of these image sequences. Our final feature vector thus consisted of 54 features.

\subsection{Dataset}

Bilateral breast MRI sagittal scans of 57 women diagnosed with breast cancer were collected at our institution from 2007-2010. The ages of the women at the time of the imaging ranged from 37 to 74 years with a mean age of 55.5 years. The women were imaged prone in a $1.5 \mathrm{~T}$ scanner (GE LX echo, GE Healthcare, or Siemens Sonata, Siemens); matrix size: $512 \times 512$; slice thickness: $2.4-4.4 \mathrm{~mm}$; flip angle: $25^{\circ}$ or $30^{\circ}$. The images were collected before and after the administration of gadodiamide (Omniscan) or gadobenate dimeglumine (MultiHance) contrast agents. Dynamic contrast enhanced images were acquired at 90 second intervals for 3 post contrast time points. The women in the dataset had estrogen receptor positive (ER+), node negative tumors, which were analyzed with the Oncotype DX prognostic gene expression assay. Oncotype DX ${ }^{\mathrm{TM}}$ is a test that was developed by Genomic Health Inc. to calculate the risk of the recurrence by measuring the expression of 21 genes in RNA from formalin-fixed paraffin-embedded (FFPE) tumor tissue samples from the primary breast cancer [9]. The final outcome of the Oncotype DX assay is a continuous recurrence score that predicts the likelihood of breast cancer recurrence in 10 years after the treatment. It has been shown that the benefit of adjuvant chemotherapy regimen starts becoming significant only in patients with an Oncotype score greater than 30 [9]. 


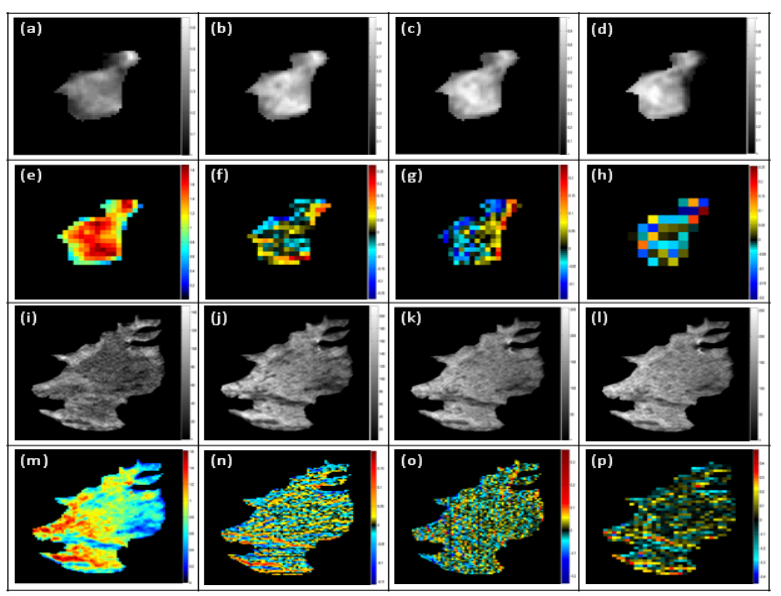

Fig. 3. The low-risk (top two rows) and high-risk (bottom two rows) contrast and the corresponding wavelet images from the first post contrast; (a)-(d), pre contrast and post contrast images of the lesion;(e) approximate wavelet coefficients at DP1;(f) horizontal wavelet coefficients at DP1;(g) vertical wavelet coefficients at DP1;(h) diagonal wavelet coefficients at DP2; (i)-(p) are similar to the above description but for high cancer recurrence risk

Therefore, from a clinical perspective, the decision of low versus high recurrence risk is very important to select women who are expected to benefit the most from adjuvant chemotherapy. In this study we tested our features for the prediction of Oncotype recurrence risk categories, where we consider patients with Oncotype score greater than 30 as high risk, and with scores less than or equal to 30 as low risk patients.

\subsection{Feature Selection and Classification}

After extracting the HetWave features, a genetic algorithm (GA)-wrapper based feature selection step is applied. GA globally looks for a subset of the features that maximize a fitness function by exploiting inheritance, mutation, selection, and crossover processes. In this study, the area under the curve (AUC) for the ROC of the training data is used as the fitness function. The population size (which specifies how many subsets are in every generation), the mutation rate, and the crossover rate are set to $100,0.05$, and 0.8 , respectively. The process of finding the optimal feature subset using the GA and the fitness function stops when it satisfies the stopping criterion; the stopping criterion is defined when the average relative change of less than 1e- 6 in the best fitness function value over 50 generations is formed.

A leave-one-out cross validation is used to evaluate the methodology. A separate feature selection for each case in leave-one-out loop is performed. The selected features are used to learn a logistic regression model to predict the recurrence risk category of women. We varied the dimensionality (number of features in the subset) from $\mathrm{K}=4$ to 12, and kept record of the features that gave the best AUC for each $\mathrm{K}$. We compared our proposed features against the following previously used features in [2]: kinetic (maximal uptake, time to peak, uptake rate, washout rate, curve shape index, 
enhancement at first post contrast time point, and enhancement ratio), textural (contrast, correlation, energy, homogeneity, entropy, variance, sum average, sum variance, sum entropy, difference in variance, difference in entropy, information measure of correlation (IMC) 1and 2, maximal correlation coefficient), and spatial enhancement variance features (maximal variance in uptake; variance in time to peak, uptake rate, and washout rate). The Genetic algorithm-wrapper based feature selection was also applied to these features and the feature subset ( $\mathrm{K}=4$ to 12) corresponding to the best AUC was selected.

\section{Results}

The comparison between the ROCs of our HetWave features versus the previously proposed features is presented in Figure 4. As shown in the figure, HetWave features provide better AUC as compared to other commonly used features. The AUC of the HetWave, standard kinetic, standard texture, and spatial enhancement variance are 0.78 , $0.69,0.64$, and 0.65 , respectively. The most frequent features selected by the feature selection technique in each leave-one-out loop are as follows: HetWave features for the mean horizontal and vertical wavelet detail coefficients of DP1 on partition 1, 2, and 3; and HetWave features for variance of horizontal and vertical wavelet detail coefficient of DP1 from partition 1. From the selected features of wavelet detail coefficients of DP1, it seems that the mean of high spatial frequency enhancement rate which is obtained from DP1 provide more relevant information for task of recurrence risk classification as compared to those of DP2 (which has coarser frequency than DP1).

Since the selected features include the HetWave features corresponding to the variance of the horizontal and vertical wavelet detail coefficients of DP1 from partition 1 , it can also be inferred that the variance of the high spatial frequency information of the partition 1 which has less local spatial entropy and variance, provides useful

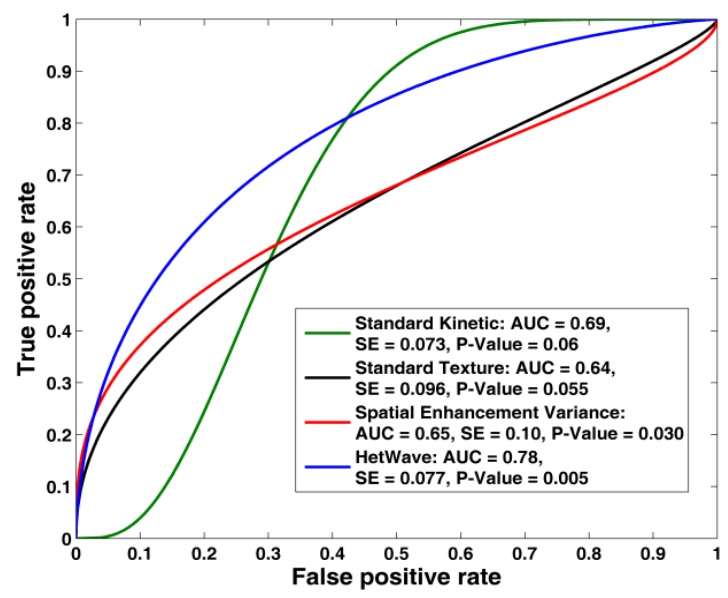

Fig. 4. ROC comparison of HetWave with other standard features 
information for this prognostic classification task. As such, capturing tumor heterogeneity using entropy-based partitioning of tumors and obtaining the spatiotemporal Information from the contrast agent uptake as well as spatial frequency information from these partitions could provide valuable information for tumor characterization.

\section{Conclusions}

In this study, heterogeneity wavelet kinetic features are introduced and applied for the task of classifying breast cancer recurrence risk. The proposed features are geared toward capturing tumor heterogeneity by partitioning the tumor into subregions, and the spatiotemporal patterns of the contrast agent and based on spatial frequency information using wavelet coefficients. An AUC of 0.78 is obtained using the proposed HetWave features which outperform other commonly used standard kinetic, texture, and spatial enhancement variance features which give AUCs of 0.69, 0.65 and 0.64 respectively.

\section{References}

1. Schnall, M.D., et al.: Diagnostic architectural and dynamic features at breast MR imaging: multicenter study. Radiology 238, 42-53 (2006)

2. Bhooshan, N., Giger, M.L., Jansen, S.A., Li, H., Lan, L., Newstead, G.M.: Cancerous breast lesions on dynamic contrast-enhanced MR images: computerized characterization for image-based prognostic markers. Radiology 254, 680-690 (2010)

3. Chen, W., Giger, M.L., Lan, L., Bick, U.: Computerized interpretation of breast MRI: investigation of enhancement-variance dynamics. Med. Phys. 31, 1076-1082 (2004)

4. Lee, S.H., Kim, J.H., Cho, N., Park, J.S., Yang, Z., Jung, Y.S., Moon, W.K.: Multilevel analysis of spatiotemporal association features for differentiation of tumor enhancement patterns in breast DCE-MRI. Med. Phys. 37, 3940 (2010)

5. Zheng, Y., Englander, S., Baloch, S., Zacharaki, E.I., Fan, Y., Schnall, M.D., Shen, D.: STEP: spatiotemporal enhancement pattern for MR-based breast tumor diagnosis. Med. Phys. 37(7), 3192-3204 (2009)

6. Agner, S., Soman, S., Libfeld, E., McDonald, M., Thomas, K., Englander, S., Rosen, M.A., Chin, D., Nosher, J., Madabhushi, A.: Textural kinetics: a novel dynamic contrastenhanced (DCE)-MRI feature for breast lesion classification. J. Digit. Imaging 24(3), 446-463 (2011)

7. Marusyk, A., Polyak, K.: Tumor heterogeneity:causes and consequences. Biochim. Biophys. Acta 1805, 105-117 (2010)

8. Yang, J., Honavar, V.: Feature subset selection using a genetic algorithm. IEEE Intelligent Systems and their Applications 13(2), 44-49 (1998)

9. Paik, S., Tang, G., Shak, S., Kim, C., Baker, J., Kim, W., Cronin, M., Baehner, F.L., Watson, D., Bryant, J., Constantino, J.P., Geyer, C.E., Wickerham, D.L., Wolmark, N.: Gene expression and benefit of chemotherapy in women with node-negative, estrogen receptorpositive breast cancer. J. Clin. Oncol. 24(23), 3726-3734 (2006)

10. Gonzalez, R., Woods, R.E.: Digital Image Processing. Prentice Hall (2007)

11. Hylton, N.: MR imaging for assessment of breast cancer response to neoadjuvant chemotherapy. Magn. Reson. Imaging Clin. N. Am. 14(3), 383-389 (2006) 